\title{
COMPRIMENTO DE ESTACA DE CAMU-CAMU COM ÁCIDO INDOLBUTÍRICO PARA A FORMAÇÃO DE MUDAS ${ }^{1}$
}

\author{
JHON PAUL MATHEWS DELGADO² \& KAORU YUYAMA ${ }^{3}$
}

RESUMO - O fruto do camu-camu chama a atenção pelo alto teor de vitamina C (877 a $6.116 \mathrm{mg} / 100 \mathrm{~g}$ de polpa). A propagação normalmente é por semente, que proporciona segregação em diferentes características de interesse comercial, enquanto a propagação vegetativa permite obter plantas de melhor uniformidade. $\mathrm{O}$ trabalho teve como objetivo avaliar o efeito do ácido indolbutírico - AIB ( 0 e $\left.200 \mathrm{mg} \mathrm{L}^{-1}\right)$, no enraizamento de estacas provenientes de ramos juvenis de camu-camu $(5 ; 10$ e $20 \mathrm{~cm}$ de comprimento, com 2 a $3 \mathrm{~cm}$ de diâmetro). As estacas de $20 \mathrm{~cm}$ de comprimento com aplicação de AIB são indicadas para a produção de mudas, cujo índice atinge $50 \%$ de mudas formadas (brotos + raiz). A utilização de AIB produziu um efeito positivo no desenvolvimento do número e comprimento de raízes. O índice de estacas enraizadas foi de $58 \%$ com a utilização de AIB e de 5\% sem AIB. Efeito negativo foi observado em relação ao número de brotos (com AIB: 1,0; sem AIB: 1,8) e estacas brotadas (com AIB: 22\%, sem AIB: 49\%). As estacas de $20 \mathrm{~cm}$ de comprimento comportam-se positivamente no número de brotos por estaca $(2,0)$ e estacas brotadas $(55 \%)$, em relação às estacas de $10 \mathrm{~cm}$ com 1,1 broto e $33 \%$, e $5 \mathrm{~cm}$ com 1,1 broto e $19 \%$.

Termos para indexação: Myrciaria dubia, enraizamento, estaquia, AIB, produção de mudas.

\section{CUTTING LENGHT OF CAMU-CAMU WITH INDOLEBUTYRIC ACID FOR CLONAL PRODUCTION}

\begin{abstract}
The fruit of camu-camu is attractive because of its high vitamin C content ( 877 to $6113 \mathrm{mg} / 100$ $\mathrm{g}$ of pulp). Its propagation normally is by seed, which segregate for different characteristics of commercial interest; hence a vegetative propagation method would allow better uniformity. This study evaluated the effect of indolebutyric acid - IBA ( 0 and $\left.200 \mathrm{mg} \mathrm{L}^{-1}\right)$ on rooting of young branches of camu-camu $(5,10$ and $20 \mathrm{~cm}$ of length and 2 to $3 \mathrm{~cm}$ in diameter). The $20 \mathrm{~cm}$ long cuttings with IBA application were most appropriate for production of camu-camu clones, with 50\% successfully formed (sprouts + root). IBA had a positive effect in the development of root number (with IBA - 12; without IBA - 1), root length (with IBA $-11 \mathrm{~cm}$; without IBA $-4 \mathrm{~cm}$ ) and rooted cuttings (with IBA $-58 \%$; without IBA $-5 \%$ ), but negative in sprout number (with IBA - 1.0; without IBA - 1.8) and cuttings sprouted (with IBA - 22\%; without IBA $-49 \%$ ). The $20 \mathrm{~cm}$ long cuttings had 2.0 sprouts and $55 \%$ cuttings sprouted, while the $10 \mathrm{~cm}$ cuttings had 1.1 sprouts and $33 \%$, and the $5 \mathrm{~cm}$ had 1.1 sprouts and $19 \%$.
\end{abstract}

Index terms: Myrciaria dubia, rooting, cuttings, AIB, clonal production.

\section{INTRODUÇÃO}

O camu-camu (Myrciaria dubia, Myrtaceae), uma fruteira nativa da Amazônia, é encontrado naturalmente nas margens dos rios, lagos e igapós, tanto em águas escuras (PETER; VÁSQUEZ, 1987; YUYAMA, 2002) como claras (YUYAMA, 2002). $\mathrm{O}$ interesse pelo fruto de camu-camu deve-se ao seu potencial em ácido ascórbico, com uma variação de 845 a $6.112 \mathrm{mg}$ em $100 \mathrm{~g}$ de polpa (YUYAMA et al., 2002).

O camu-camu está sendo domesticado no Brasil em solos de terra firme (YUYAMA, 2002) e, no Peru, em áreas da planície inundável da Amazônia (PENN, 2006).
No Peru, o camu-camu vem sendo plantado em solos de várzea (solos inundados de dois a três meses durante o ano). Os $75 \%$ das plantações foram instalados em espaçamento de $3 \times 3 \mathrm{~m}$ (1.111 plantas/ ha) (PENN, 2006). Este espaçamento, aos 10 anos, limita a produção por planta. Neste sentido, o raleio para deixar os plantios em espaçamento de $6 \times 3 \mathrm{~m}$ é importante para a melhoria da produção. Porém, muitas plantas que serão eliminadas têm características de interesse pelo produtor. Neste sentido, para o máximo aproveitamento destas matrizes, através da propagação por estaquia, seriam usados também os ramos mais grossos, que aumentariam a taxa multiplicativa em plantas de onze anos. 
Normalmente, o camu-camu é propagado por semente, porém apresenta segregação em diferentes características de interesse agronômico (PINEDO et al., 2001; YUYAMA, 2002), sugerindo-se a necessidade da propagação vegetativa para a manutenção das características desejáveis das plantas-matrizes (HARTMAN et al., 1997).

A capacidade de uma estaca emitir raízes está em função de fatores endógenos e das condições ambientais proporcionadas ao enraizamento. Entre tais fatores, os fitorreguladores são de importância fundamental, destacando-se formação de auxinas que apresentam o maior efeito na formação de raízes em estacas (FACHINELLO et al., 2005).

O camu-camu, para ser propagado por estaquia, deve utilizar-se de ramos juvenis provenientes de posições basais, com diâmetros de $8 \mathrm{~mm}$, em imersão de 12 a 14 horas em solução de ácido naftalenoacético (ANA) (200 ppm), para conseguir 90\% de enraizamento (PEREIRA, 2002).

Arévalo (2003) recomenda usar estacas das posições basais com diâmetros maiores de $2,5 \mathrm{~cm}$ para obter $60 \%$ de enraizamento.

Assim mesmo, em camu-camu, a utilização de ramos lenhosos e a aplicação de fitorreguladores, como as auxinas, têm sido utilizadas por muitos pesquisadores com estacas com comprimentos de 20 a $25 \mathrm{~cm}$, e usando diâmetro que variam de 0,8 a $2 \mathrm{~cm}$ (SANTANA, 1998; AZEVEDO, 1999; PEREIRA, 2002; ARÉVALO, 2003; OLIVA, 2005), cujo índice de enraizamento atinge entre 50 e $90 \%$.

No entanto, o material de propagação utilizado por diferentes pesquisadores (SANTANA, 1998; PEREIRA, 2002; AREVALO, 2003), coletado nas posições basais (ramos verticais), é escasso, tendo em vista que o objetivo é produzir um grande número de mudas por estaquia.

Lima et al. (2006) verificaram em Malpighia emarginata, que a produção de mudas por estacas é determinada pela maior quantidade de reservas nutritivas presentes em estacas de $20 \mathrm{~cm}$ de comprimento, comparado àqueles de $15 \mathrm{e} 10 \mathrm{~cm}$ de comprimento.

Do mesmo modo, Hartman et al.(1997) indicam que a maior quantidade de reservas nutritivas é um dos fatores responsáveis pelo enraizamento.

A propagação por estaquia em camu-camu está orientada à propagação de plantas-matrizes promissoras para a formação de jardins clonais, devido a limitações de material multiplicativo por planta (YUYAMA, comunicação pessoal).

Diante desse contexto, este trabalho teve como objetivo desenvolver uma técnica de propagação por estaquia para aumentar a taxa multiplicativa do camu-camu, aproveitando as plantas provenientes do raleio, estudando o comprimento das estacas tratadas com AIB.

\section{MATERIAIS E MÉTODOS}

O trabalho foi conduzido no IIAP (Instituto de Investigación de la Amazonía Peruana), localizado em Iquitos-Peru, no período de agosto a novembro/2005. O material propagativo utilizado foram estacas lenhosas (ramos verticais), oriundo de plantas-matrizes com onze anos de idade e cultivadas em solos de várzea.

Os ramos foram coletados em agosto, pela manhã, dois meses após o período da estiagem das águas. As plantas-matrizes encontravam-se em fase vegetativa.

Retiraram-se a partir das posições basais, as médias dos ramos para realizar a segmentação em estacas de 5; 10 e $20 \mathrm{~cm}$ de comprimento, com diâmetro aproximado de 2 a $3 \mathrm{~cm}$, descartando-se a parte apical. Posteriormente, as estacas foram imersas, até $50 \%$ do comprimento da estaca, por 48 horas, em uma solução com ácido indolbutírico (AIB), nas concentrações de $200 \mathrm{mg} \cdot \mathrm{L}^{-1}$. O AIB foi dissolvido em hidróxido de potássio $\left(\mathrm{KOH}_{5} \mathrm{~N}\right)$, na proporção $1 \mathrm{~g}$ de AIB para 1 mililitro do $\mathrm{KOH}_{5} \mathrm{~N}$, e o restante do volume, completado com água destilada. Para a concentração-testemunha, foi utilizada apenas água.

O plantio foi realizado em uma caixa de madeira de 1 x $5 \mathrm{~m}$, com $30 \mathrm{~cm}$ de altura, em condições ambientais, contendo como substrato húmus originado de serragem, sendo a irrigação realizada diariamente, por aspersão de $30 \mathrm{~min}$.

O delineamento experimental foi em blocos ao acaso, com arranjo fatorial de $2 \times 3$, com cinco repetições e 30 estacas por parcela. Sendo os fatores: concentrações de AIB ( 0 e 200 mg.L $\left.\mathrm{L}^{-1}\right)$ e comprimento de estaca $(5 ; 10$ e $20 \mathrm{~cm})$.

Após o período de 90 dias, as características analisadas foram: porcentagem de estacas enraizadas, porcentagem de mudas formadas (broto + raiz), número médio das raízes mais desenvolvidas, comprimento da maior raiz, porcentagem de estacas brotadas e número médio de brotos.

Os dados foram submetidos à análise da variância, pelo teste F, e, quando significativos, comparadas as médias pelo teste de Tukey, a 5\% de significância. Os dados expressos em porcentagem foram transformados em arco-seno da raiz de $\mathrm{x}+0,5 / 100$. 


\section{RESULTADOS E DISCUSSÃO}

O uso de AIB aumentou a porcentagem de estacas enraizadas (Tabela 1). O pouco enraizamento das estacas sem AIB foi associado possivelmente ao baixo teor de auxinas em que foram encontradas as estacas (HARTMAN et al., 1997). Resultados similares encontrou Azevedo (1999), que trabalhou com estacas de camu-camu tratadas com 150 a 1.500 $\mathrm{mg} \mathrm{L}^{-1}$ de AIB, encontrando uma media de $57 \%$ de enraizamento. Não obstante, os resultados diferem de Oliva (2005), que obteve $80 \%$ de enraizamento em estacas de camu-camu, que também utilizou $200 \mathrm{mg}$ $\mathrm{L}^{-1}$ de AIB em estacas de $2 \mathrm{~cm}$ de diâmetro e $20 \mathrm{~cm}$ de comprimento, coincidindo com Pereira (2002), que obteve em camu-camu $90 \%$ de enraizamento, utilizando $200 \mathrm{mg} \mathrm{L}^{-1}$ de ácido naftalexoacético ANA, em estacas de $8 \mathrm{~mm}$ de diâmetro e $25 \mathrm{~cm}$ de comprimento. As condições de estufa em que trabalharam os outros pesquisadores, distintas do experimento que esteve exposto ao ambiente, possivelmente minimizaram o estresse hídrico das estacas, refletindo em maiores enraizamentos (HARTMAN et al., 1997). O AIB também aumentou a porcentagem de mudas formadas. Porém, diminuiu o número de brotos e a porcentagem de brotação (Tabela 1). As estacas de camu-camu tratadas com fitorreguladores iniciam a formação de raiz primeiro que a formação de brotos, utilizando os nutrientes da estaca para o desenvolvimento de raízes, ao invés de brotos (OLIVA, 2005; SANTANA, 2009). Vários trabalhos de propagação por estaquia de camu-camu não mencionam a porcentagem de formação de mudas (AZEVEDO, 1999; AZEVEDO; NAGÃO, 1998; OLIVA, 2005; PEREIRA, 2002; YUYAMA, 2001), porém esta variável é importante para determinar a quantidade de mudas disponível para o transplante a campo definido. Neste sentido, depois de terminado o ensaio, as estacas enraizadas foram acondicionadas para observar se completavam a formação da parte aérea, mas não foi observado brotação. Coincidindo com Santana (1998), que uma estaca com raiz e sem broto não garante a formação da parte aérea depois de terminada a etapa de propagação do camu-camu.

A estaca com $20 \mathrm{~cm}$ de comprimento foi maior na porcentagem de mudas formadas, em comparação com as estacas de 5 e $10 \mathrm{~cm}$, embora o comprimento de estaca não tivesse diferença significativa na porcentagem de estacas enraizadas e no número e comprimento de raízes (Tabela 1). O maior comprimento de estacas possivelmente tenha relação direta com a maior disponibilidade de nutrientes armazenadas, contribuindo diretamente com o maior fornecimento de nutrientes para a formação de brotos e raízes
(HARTMAN et al., 1997). Assim mesmo, o maior volume de estacas de camu-camu está relacionado positivamente com maior enraizamento (AREVALO, 2003). Lima et al. (1992) também encontraram, nos comprimentos de estacas de 15 e $20 \mathrm{~cm}$, maior porcentagem de formação de mudas, trabalhando com três comprimentos de estacas de Malpighia glabra, com 10; 15 e $20 \mathrm{~cm}$ de comprimento. Um maior comprimento de estaca também é positivo no número de brotos por estacas (Tabela 1). A formação de múltiplos caules desde a base em plantas de camu-camu está relacionada positivamente com a arquitetura de copa tipo taça, sendo este desejável, por estar relacionado à maior produção de fruta (YUYAMA, 2002).

Por outro lado, estacas com $20 \mathrm{~cm}$ de comprimento e tratadas com AIB incrementaram a porcentagem de mudas formadas, superando os comprimentos de 10 e $5 \mathrm{~cm}$. Estacas em que não foram aplicadas AIB obtiveram as menores porcentagens de mudas formadas, independentemente do comprimento (Tabela 2). Fachinello et al. (1995) indicam que o enraizamento das estacas depende de fatores endógenos, tais como a quantidade de hormônios e reservas de carboidratos disponíveis nas estacas, o que poderia ter influenciado de forma positiva no logro de mudas nas estacas de $20 \mathrm{~cm}$ com AIB. 
TABELA 1 - Número médio de raízes, comprimento maior de raiz, porcentagem de enraizamento, porcentagem de mudas formadas, número médio de brotos e porcentagem de brotação em diferentes comprimentos de estacas de camu-camu e concentração de AIB.

\begin{tabular}{ccccccc}
\hline & $\begin{array}{c}\text { Número } \\
\text { médio de } \\
\text { raízes }\end{array}$ & $\begin{array}{c}\text { Comprimento } \\
\text { da maior raiz } \\
(\mathbf{c m})\end{array}$ & $\begin{array}{c}\text { Enraizamento } \\
\mathbf{( \% )}\end{array}$ & $\begin{array}{c}\text { Mudas } \\
\text { formadas } \\
\mathbf{( \% )}\end{array}$ & $\begin{array}{c}\text { Número } \\
\text { médio de } \\
\text { brotos }\end{array}$ & $\begin{array}{c}\text { Brotação } \\
\mathbf{( \% )}\end{array}$ \\
\hline $\begin{array}{c}\text { Comprimento } \\
(\mathrm{cm})\end{array}$ & & & & & & \\
20 & $7,6^{\mathrm{ns}}$ & $8,0^{\mathrm{ns}}$ & $39 \mathrm{~ns}$ & $28 \mathrm{a}$ & $2,0 \mathrm{a}$ & $55 \mathrm{a}$ \\
10 & 6,1 & 7,0 & 27 & $8 \mathrm{~b}$ & $1,1 \mathrm{~b}$ & $33 \mathrm{~b}$ \\
5 & 5,7 & 7,7 & 30 & $7 \mathrm{c}$ & $1,1 \mathrm{~b}$ & $19 \mathrm{~b}$ \\
\hline $\mathrm{AIB}\left(\mathrm{mg} \cdot \mathrm{L}^{-1}\right)$ & & & & & & \\
0 & $1 \mathrm{~b}$ & $4 \mathrm{~b}$ & $5 \mathrm{~b}$ & $5 \mathrm{~b}$ & $1,8 \mathrm{a}$ & $49 \mathrm{a}$ \\
200 & $12 \mathrm{a}$ & $11 \mathrm{a}$ & $58 \mathrm{a}$ & $24 \mathrm{a}$ & $1,0 \mathrm{~b}$ & $22 \mathrm{~b}$ \\
\hline $\mathrm{CV}(\%)$ & 28 & 46 & 40 & 55 & 36 & 27 \\
\hline
\end{tabular}

Letras minúsculas distintas na coluna, para cada variável, diferem entre si, pelo teste de Tukey, a 5\% de significância. ${ }^{\text {ns }}$ Não houve diferença significativa.

TABELA 2 - Relação do comprimento de estacas de camu-camu e concentração de AIB na porcentagem de mudas formadas.

\begin{tabular}{ccc}
\hline & \multicolumn{3}{c}{ Mudas formadas (\%) } \\
\cline { 2 - 3 } Comprimento de estaca (cm) & \multicolumn{2}{c}{ AIB (mg. $\left.\mathbf{L}^{-1}\right)$} \\
\cline { 2 - 3 } & $\mathbf{2 0 0}$ & $\mathbf{0}$ \\
\hline 5 & $9 \mathrm{bA}$ & $4 \mathrm{aA}$ \\
10 & $11 \mathrm{bA}$ & $5 \mathrm{aA}$ \\
20 & $50 \mathrm{aA}$ & $6 \mathrm{aB}$ \\
\hline
\end{tabular}

Letras minúsculas distintas na coluna e maiúsculas na linha, para cada variável, diferem entre si, pelo teste de Tukey, a 5\% de significância.

\section{CONCLUSÕES}

O melhor resultado na produção de mudas de camu-camu, via técnica de propagação por estaquia, é obtido utilizando a concentração de $200 \mathrm{mg} . \mathrm{L}^{-1} \mathrm{de}$ AIB em estacas de $20 \mathrm{~cm}$ de comprimento.

\section{AGRADECIMENTOS}

Ao projeto "Herramientas para el Mejoramiento Genético del camu-camu en Restingas / Instituto de Investigaciones de La Amazonía Peruana (IIAP) - Imnovación para el Agro Peruano (INCAGRO)", pelos recursos financeiros ao projeto. Ao Blgo Msc. César Delgado Vásquez, coordenador do projeto, pelo incentivo e apoio permanente às pesquisas realizadas em camu-camu.

\section{REFERÊNCIAS}

ARÉVALO L. Evaluación de métodos de clonación de camu-camu (Myrciaria dubia), mediante estacas. 2003. 75f. Monografia (Trabalho Graduação em Agronomia) - Facultad de ciencias agronómicas, Universidad Nacional de la Amazonía Peruana, Iquitos, 2003.

AZEVEDO, D.M. Efeito do ácido indolbutírico (AIB) e substratos sobre o enraizamento de estacas de camu-camu (Myrciaria dubia (H.B.K.) McVaugh). 1999. 40f. Monografia (Trabalho de Graduação em Agronomia) - Faculdade de ciências Agronômicas, Universidade Federal do Amazonas, Manaus, 1999. 
AZEVEDO, D.M.; NAGÃO, E.O. Efeito de ácido indolbutírico (AIB) e substratos sobre o enraizamento de estacas de camu-camu (Myrciaria dubia (H.B.K.) McVaugh). 1998. 42f. Relatório Final CNPq (Agronomia) - Faculdade de Ciências Agronômicas, Universidade Federal do Amazonas, Manaus, 1998.

FACHINELLO, J. C.; HOFFMANN, A.; NACHTIGAL, J. C.; KERSTEN, E.; FORTES, G. R. de L. Propagação de plantas frutíferas de clima temperado. 2. ed. Pelotas: UFPel, 1995. 178p.

HARTMANN, H.T.; KESTER, D.E.; DAVIES JR., F.T.; GENEVE, R.L. Plant propagation, principles and practices. 6. ed. New Jersey: Upper Saddle River/Prentice Hall, 1997. 770p.

LIMA, H.T.; ALMEIDA, F.A.C.; ALMEIDA, F.C.G. Estudo sobre o enraizamento de estacas de acerola (Palpighia glaba L.). Revista Brasileira de Fruticultura, Jaboticabal, v.14, n.1, p.7-13, 1992.

OLIVA, C. Efecto de fitoreguladores enraizantes y la temperatura en el enraizamiento de estacas de myrciaria dubia (HBK) mc vaugh,camu camu arbustivo, en ucayali-Perú. Folia Amazónica, Iquito, v.14, n.2, p.19-25, 2005.

PENN, J.W.JR. The cultivation of camu camu (Myrciaria dubia): A tree planting programme in the Peruvian amazon. Forests, Trees and Livelihoods, Great Britain, v.16, p.85-101, 2006.
PEREIRA, B.G. Produção de mudas de camucamu por estaquia utilizando ramos provenientes de diferentes tipos e posição da planta. 2002. 53f. Monografia (Ciências Agrárias) - Faculdade de Ciências Agronômicas, Universidade Federal do Amazonas. Manaus, 2002.

PETERS, C.M.; VASQUEZ, A. Estudios ecológicos de Camu-Camu ( Myrciaria dúbia ). I-Producción de frutos en poblaciones naturales. Acta Amazonica, Manaus, v.17, n.4, p.161-173, 1987.

PINEDO, P.M.; RIVA, R.R.; RENGIFO, S.E.; DELGADO, V.C.; VILLACRÉS, V.J.; GONZÁLEZ, C.A.; INGA, S.H.; LÓPEZ, U.A.; FARROÑYY, P.R.; VEGA, V.R.; LINARES, B.C. Sistema de producción de camu-camu en restinga. Iquitos: Instituto de Investigación de la Amazonía Peruana, 2001.141p.

SANTANA, S.C. Propagação de Camu camu (Myrciaria dubia (H.B.K) Mc Vaugh) por estaquia. Biotecnología Ciência \& Desenvolvimento. Boa vista, v.29, p.165-171.1998. Disponível em: $<$ http:// www.biotecnologia.com.br/bio29/camucamu.asp $>$. Acesso em: 01 fev. 2009

YUYAMA, K. Livro de resultados dos Projetos de Pesquisa Dirigida (PPDs). Domesticação de germoplasma de camu-camu (Myrciaria dubia (H.B.K.) McVaugh). Manaus: Instituto de Pesquisa da Amazônia, 2002. p.149-153.

YUYAMA, K.; AGUIAR, J.P.L.; YUYAMA, L.K.O. Camu-camu: Um fruto fantástico como fonte de vitamina C. Acta Amazonica, Manaus, v.32, n.1, p.169-174, 2002. 